\title{
QUANTIFICATION OF CSF VELOCITY THROUGH THE NARROWEST POINT IN AQUEDUCT OF SYLVIA FOR NORMAL AND NORMAL PRESSURE HYDROCEPHALUS PATIENT BY CFD ANALYSIS
}

\author{
THALAKOTUNAGE A. H, THUNYASETH SETHAPUT \\ Sirindhorn International Institute of Technology, Thammasat University, Pathum Thani, Thailand \\ Email: thunyaseth@siit.tu.ac.th
}

Received: 23 Apr 2016 Revised and Accepted: 29 May 2016

\begin{abstract}
The aim of this comprehensive study is to quantify the velocity variation of cerebrospinal fluid (CSF) for narrowest point in aqueduct of Sylvia (AqSylv) of normal patients and normal pressure hydrocephalus (NPH) patient by corresponds to its concave shapes of anteriorly and inferiorly. T1-weighted 3-T magnetic resonance images (MRI) of the head in DICOM (Digital Imaging and Communications in Medicine) format were taken from three controlled patients whose were admitted to Thammasat Hospital, Thailand. Patients were 29 to 52 y of age with two normal patients and one (NPH) patient. DICOM files were three-dimensionally reconstructed by using 3D slicer software, and geometric information of an aqueduct for all three cases was noted. Solid models of the aqueduct for both normal patient and NPH condition were developed based on the geometric information. Computational fluid dynamics (CFD) were analyzed to quantify the CSF velocity variation throughout the narrowest point of the aqueduct for both cases, i.e. normal and NPH condition. Retrospective results of "mathematical model for dynamics of CSF through the aqueduct of Sylvia based on an analogy of arterial dilation and contraction" were used as initial data for ANSYS CFX analysis. The results showed the CSF flow through the aqueduct in a pulsatile pattern in both cases. At the narrowest point of the aqueduct, amplitude of peak CSF velocity for NPH patients was significantly higher than that of normal patient. CSF velocity variation throughout the aqueduct co-relates with the pressure gradient inside the aqueduct and increased in the third ventricle direction.
\end{abstract}

Keywords: Aqueduct of Sylvia, CFD Analysis, 3D reconstruction, CFX, CSF, MRI, NPH, Hydrocephalus

(C) 2016 The Authors. Published by Innovare Academic Sciences Pvt Ltd. This is an open access article under the CC BY license (http://creativecommons.org/licenses/by/4.0/] DOI: http://dx.doi.org/10.22159/ijpps.2016v8s2.15222.

\section{INTRODUCTION}

The aqueduct of Sylvia (AqSylv) is a tube which connects the third and fourth ventricles. Investigations of Lindgren and DI Chiro [1] revealed that diameter of the aqueduct was varying throughout its length and showed a shape of a swan neck. Characteristics and parameters of AqSylv were more important since it was a passage allowed CSF to flow from third to fourth ventricles. Any abnormalities in dimensions of the aqueduct could lead to the malfunctioning in the motion of CSF. CFS secrets from the choroid plexus in lateral, third and fourth ventricles and enters in the subarachnoid spaces (SAS). Fig. 1 displays the ventricle system and CSF pathway of the human brain. Since cranial volume is constant, CSF absorption by arachnoid granulations into venous circulation is inevitable circumstance and the absorption rate is linearly related to the intracranial pressure (ICP) [2]. Monro-Kellie's doctrine represents the compensation and auto-regulation process of cerebral blood and CSF volumes in cranial space. CSF is a colorless liquid [3] and permeates from the choroid plexus at a rate of 0.2-0.7 $\mathrm{ml} /$ minute. The ventricular system accommodates $20 \%$ of CSF volume out of its total volume of $125-150 \mathrm{ml}$ at a time. Dominant percentage of CSF manufacturers in lateral, third ventricles and moves as a bulk flow through the AqSylv to the fourth ventricle [4]. Any obstruction in AqSylv would bring about a blockage of CSF continuum flow and could lead to enlargement of ventricles due to the CSF trapping inside the lateral and third ventricles, which is symptomatic of congenital or obstructive hydrocephalus $[5,6]$ Aqueductal stenosis narrow the cross-sectional area of AqSylv and create a resistance to the CSF flow. Malfunctioning in any CSF motion would increase the volume of the ventricles by resulting in the higher built-in pressure inside the ventricle system and enlarging towards the skull [7]. Scientist considered aqueductal stenosis was a root cause of non-communicating hydrocephalus, as the obstruction in the aqueduct would result in the accumulation of CSF in the ventricles. In the communicating hydrocephalus, the lateral ventricles and medial parts of the temporal lobes expand owing to accumulated CSF inside and compress the aqueduct as a consequence of volume compensation in the skull. Subsequently, the pressure inside the fourth ventricle declined and caused the aqueduct to close more tightly. These phenomena were also illustrated as aqueductal stenosis, a root cause of hydrocephalus [8].

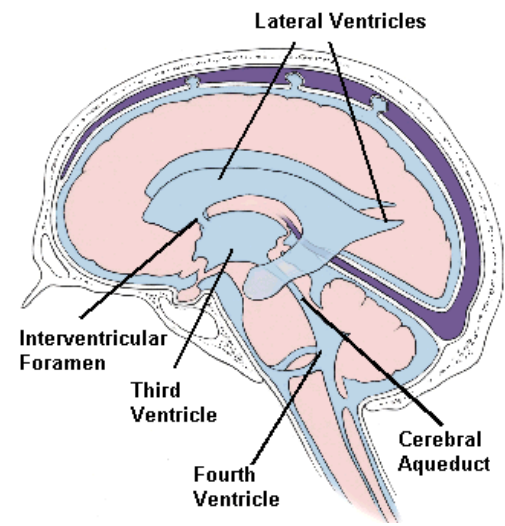

Fig. 1: Ventricular system and cerebral spinal fluid pathway [9]
Nomenclature
AqSylv- Aqueduct of Sylvia
ICP- Intracranial Pressure
CFD- Computational Fluid Dynamics
MRI- Magnetic Resonance Imaging
CSF- Cerebrospinal Fluid
NPH- Normal Pressure Hydrocephalus

DICOM- Digital Imaging and Communications in Medicine 


\section{MATERIALS AND METHODS}

CFD was analyzed to quantify the velocity variation of CSF through the AqSylv. MRI DICOM of the aqueduct was taken in particular two normal patients and one NPH patient. DICOM files were exported to the 3D Slicer software and 3D models were generated. Geometric information of an aqueduct which corresponded to the normal and NPH patients was noted. Measured mean morphologic of the ventricular system was, width of lateral ventricles $30 \mathrm{~mm}$; length of aqueduct $14.1 \mathrm{~mm}$; diameter of the narrowest point of the aqueduct for a normal patient $0.9 \mathrm{~mm}$; inclination of aqueduct relates to the third and fourth ventricles is $26^{\circ}$ and $18^{\circ}$ respectively [10] diameter of the narrowest point of the aqueduct for hydrocephalus patient were reported as $0.5 \mathrm{~mm}$ and we assumed it as $0.6 \mathrm{~mm}$ for NPH patient. Solid models of the aqueduct were constructed for both cases by considering the anatomical shape of concave anteriorly and inferiorly. Computed solid models by using SolidWorks $(\subset)$ were exported to the ANSYS CFX to generate the mesh, and set up the boundary conditions. In addition to the retrospective computational fluid analysis had done by other researchers based on the rigid wall domain for the aqueduct, we considered aqueduct wall as a deformable membrane. Properties that we used are showing in table 1. Navier-Stokes equations applied as the continuity of CSF throughout the aqueduct. Several assumptions were made such as CSF is incompressible, viscous, homogenous and Newtonian fluid. Considering aqueduct wall as deformable membrane was more important, whereas it influenced the pressure variation by acting as elastic buffering chamber due to its elastic properties $[11,12]$.

Table 1: Characteristic properties of CSF and tissues

\begin{tabular}{ll}
\hline Property & Value \\
\hline Young Modulus of Ventricles & $2,100 \mathrm{~N} / \mathrm{m}^{2}$ \\
Young Modulus of SAS & $3,500 \mathrm{~N} / \mathrm{m}^{2}$ \\
Density of CSF, $\rho_{\mathrm{f}}$ & $1,004-1,007 \mathrm{~kg} / \mathrm{m}^{3}$ \\
Viscosity of CSF, $\mu_{\mathrm{CSF}}$ & $10^{-3} \mathrm{Pas}$ \\
Ventricular Tissue Spring Elasticity, $\mathrm{k}_{\mathrm{e}}$ & $8 \mathrm{~N} / \mathrm{m}(\mathrm{Normal})$ \\
Brain Tissue Dampening, $\mathrm{k}_{\mathrm{d}}$ & $0.35 \times 10^{-3}(\mathrm{Ns}) / \mathrm{m}$ \\
Specific Gravity of CSF & 1.007 \\
\hline
\end{tabular}

\section{Former studies}

Former study results of "Mathematical model for dynamics of CSF through the AqSylv based on an analogy of arterial dilation and contraction" [13] used as the initiation condition of CFX analysis, and its resulted velocity profile of CSF through the aqueduct used CFX as an expression to initial conditions. Fig. 2 shows the CSF velocity profile throughout the aqueduct, which we have obtained from our former study. The upper graph shows the displacement of ventricular tissues corresponds to the arterial wall displacement during the cardiac cycle. The vertical axis denotes the millimeter and horizontal axis denotes R-R cardiac intervals. The yellow line is the cardiac pulse. " $\mathrm{X}$ " and "O" dotted lines represent the displacement of blood wall and ventricular tissue, respectively. The lower graph shows the CSF velocity proportional to the ventricular tissue displacement, and CSF flow is synchronized with cardiac pulsation in making a small phase shift.

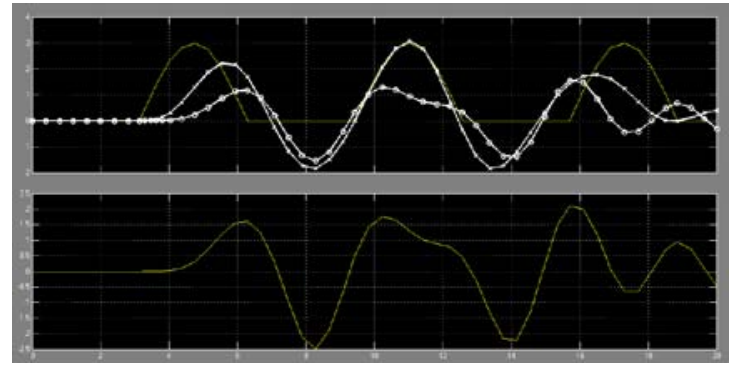

Fig. 2: CSF velocity profile through the aqueduct with respect to ventricular tissue displacement. CSF velocity in terms of "cm/sec" in term of cardiac R-R interval [13]

\section{D reconstruction and geometric information}

DICOM files were imported to 3D slicer software to do the volume rendering, and present as CT-coronary Artery view to investigate the dimensions and geometries of the ventricular system. Fig. 3 shows threedimensionally reconstructed models of NPH patient's ventricle system.

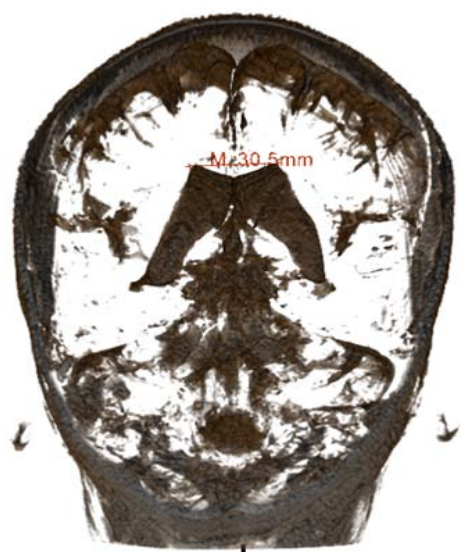

Fig. 3: 3D reconstructed ventricle system of NPH patient and geometric measurements

\section{Solid models of aqueduct of sylvia}

Solid models were built by using Solid Works@ Premium 2015. The length of the aqueduct for both cases was considered as $14.1 \mathrm{~mm}$ and the narrowest point was placed $8.5 \mathrm{~mm}$ distances from third ventricle side of the solid model. The length of the narrowest point of a normal patient's aqueduct was $1.5 \mathrm{~mm}$ and $2.7 \mathrm{~mm}$ for NPH patient in the axial direction of the aqueduct. The diameter of the narrowest point of the aqueduct has been considered as $0.9 \mathrm{~mm}$ and $0.6 \mathrm{~mm}$ for the normal patient and NPH patient correspondingly. Fig. 4 (a) and (b) shows the solid models of the aqueduct of Sylvia for the normal patient and NPH patient correspondingly.

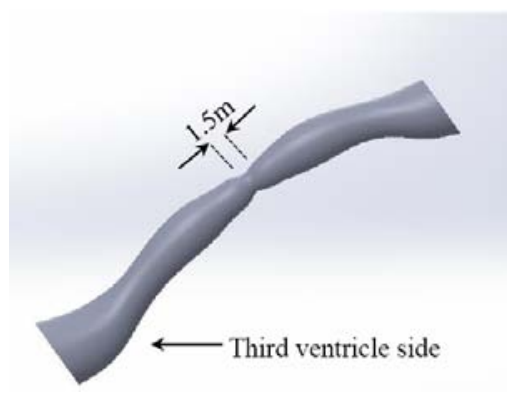

(a)

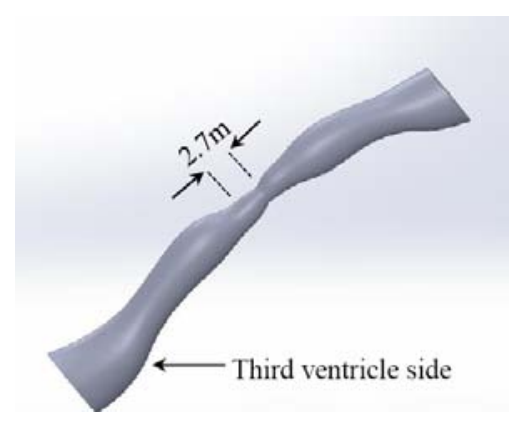

(b)

Fig. 4: Solid model of aqueduct of Sylvia for (a) normal patient and (b) NPH patient 
For both analyses, CSF concerned as water and aqueduct wall concerned as solid elastic material by assigning brain tissue elastic properties. Elasticity of the aqueduct wall changed to $6000 \mathrm{~Pa}$ and $1500 \mathrm{~Pa}$ [14] for normal patient and NPH patient respectively (Simulations were done by considering same wall elasticity for normal and NPH cases, there were no significant velocity distribution change due to the compensation of pressure by elastic property of the wall of aqueduct's narrow point). Initial boundary conditions were concerned as the same for both analysis, reference pressure of the third ventricle was $10 \mathrm{mmHg}$. Velocity profile derived from the retrospective numerical analysis was used as a mathematical expression of both cases and initial CSF input velocity into aqueduct from the third ventricle was set as defined expression (defined equation for CSF velocity profile was in our previous studies [13]). Defined mathematical expression in CFX was on one cycle of CSF pulsatile velocity. In order to get more accurate results, considered pulsatile cycle was divided into 16-time steps.

Navier-Stokes equation for the motion of CSF can be written as refer to "(1)". Where $u$ is fluid velocity, $R_{e}$ is Reynolds number, $f$ is a force, and $p$ is the mean of the three normal stresses.

$$
\begin{array}{r}
\frac{D u}{D t}=-\nabla p+f+\frac{1}{R_{e}} \nabla^{2} u \\
R_{e}=\frac{U L}{v}
\end{array}
$$

Venturi effect can be illustrated as refer to " $(2)$
$P_{\text {Third Ventricle }}-P_{\text {Narrowest Place }}=\frac{\rho}{2}\left(v_{2}^{2}-v^{2}{ }_{1}\right)$

Where, ${ }^{v_{1}}$ is the CSF velocity of third ventricle side and ${ }^{v_{2}}$ is the CSF velocity at the narrowest point of the aqueduct.

\section{RESULTS AND DISCUSSION}

CFD results showed that descending velocity gradient exists along the third ventricle direction and ascending pressure gradient exists in third ventricle direction from the narrowest point of the aqueduct. The results agree with the investigations of Jeong Hyun Lee et al. [15] which noted CSF velocity tendency to increase its velocity from the superior to the inferior aqueduct. At the narrowest point of the aqueduct, the maximum CSF velocity of normal patient reached to $31.5 \mathrm{~cm} / \mathrm{s}$ as shown in fig. 5(a). But for a NPH patient it reached to $51.7 \mathrm{~cm} / \mathrm{s}$ as shown in fig. 5(b). After that, velocity variation of CSF through the narrowest point of the aqueduct for one $\mathrm{R}-\mathrm{R}$ cycle was plotted which was based on the same input conditions on CFD analysis. Fig. 6(a) and fig. 6(b) show the CSF velocity profile at the narrowest point of the aqueduct of Sylvia for one R-R cardiac cycle of a normal patient and NPH patient correspondingly. At the narrowest point of the aqueduct, Results were explicitly shown that CSF mean velocity of NPH patient was higher than that of a normal patient. In addition, the amplitude of peak velocity of CSF for NPH patient was significantly higher than that of CSF for the normal patient as well.
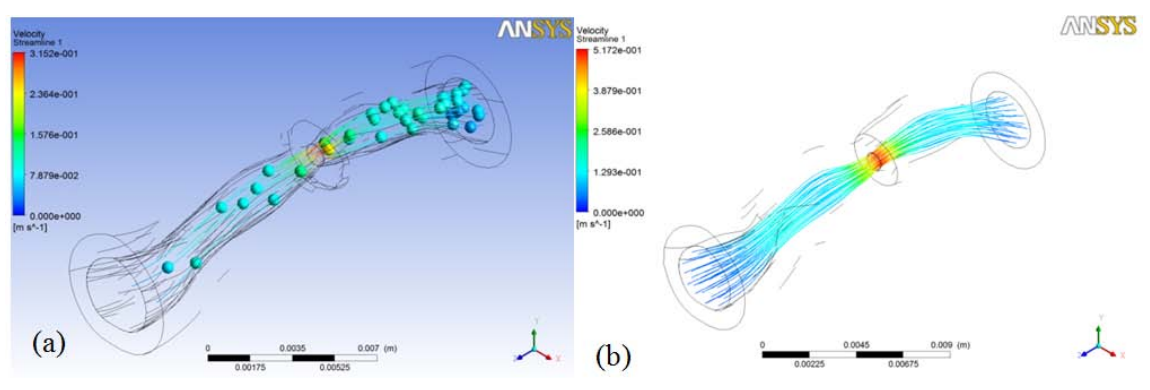

Fig. 5: CSF velocity variation through the aqueduct of Sylvia for (a) normal patient and (b) NPH patient

\section{CONCLUSION}

According to the Venturi effect, the lowest pressure exerted on the place of maximum velocity occurred at a tube of fluid flows. Same phenomena happened in the aqueduct of Sylvia where lowest pressure and highest velocity of CSF existed on the narrowest point. At the narrowest point of the aqueduct, CSF pressure of NPH patient was lower than CSF pressure of a normal patient. But, the pressure gradient of NPH patient along the third ventricle side was higher than that of the normal patient along the third ventricle side. Under the condition that the aqueduct wall elasticity was significantly low and could not compensate the pressure increment, the third ventricle would enlarge as a consequence. According to the results, we concluded that ventricular enlargements might result from the reduction of cross sectional area or obstruction at the narrow point of the aqueduct and also the reduction of wall elastic property at the narrowest point.

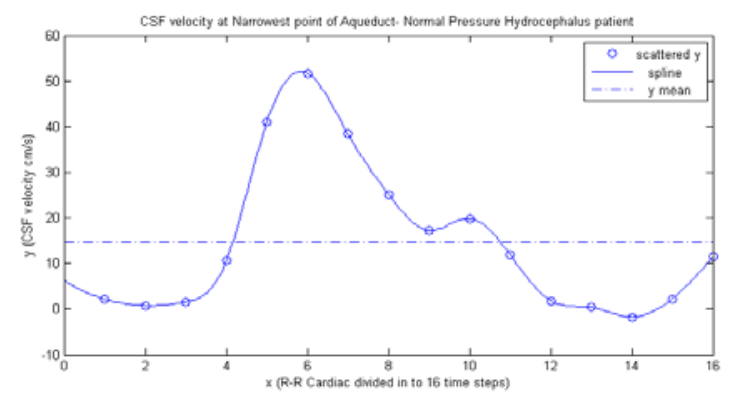

(a)

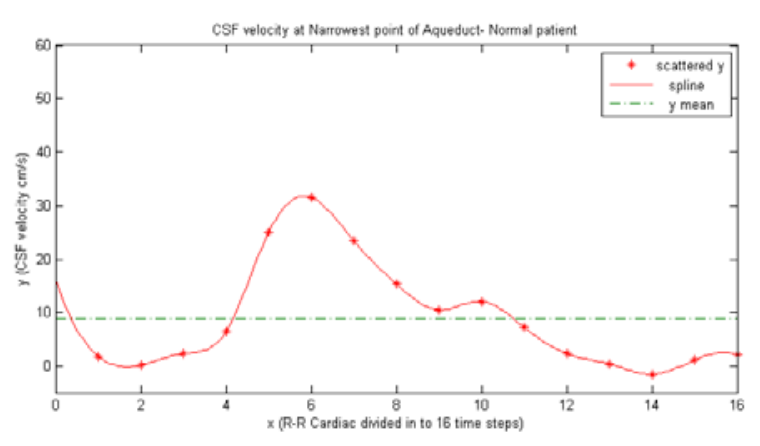

(b)

Fig. 6: CSF velocity variation through the narrowest point of the aqueduct of Sylvia for (a) normal patient and (b) NPH patient

\section{ACKNOWLEDGMENT}

We would like to convey our sincere gratitude to Dr. Varalee Mingkwansook, Ph. D, at Thammasat hospital and Assoc. Prof. Orasa Chawalparit of Mahidol University for giving MRI data and information to carry out our research. This research was supported in part by Sirindhorn International Institute of Technology, Thammasat University, grant SIIT2014-YRG5.

\section{CONFLICT OF INTERESTS}

Declared none 


\section{REFERENCES}

1. E Lindgren, G Di Chiro. The roentgenologic appearance of the aqueduct of sylvius. Acta Radiol 1953;39:117-25.

2. Anthony M Avellino, MD Benjamin S, Carson SR MD. Increased intracranial pressure. Current Management in child Neurology. Third edition. BC Decker Inc; 2009. p. 500.

3. Nafiseh Masoumi, Dariush Bastani, Siamak Najarian, Fariba Ganji, Farhad Farmanzad, Amir Saeed Seddighi. Mathematical modeling of CSF pulsatile hydrodynamics based on fluid-solid interaction. IEEE Trans Biomed Eng 2010;57:1255-63.

4. Greitz D. Cerebrospinal fluid circulation and associated intracranial dynamics. A radiologic investigation using MR imaging and radionuclide cisternography. Acta Radiol Suppl 1993;386:1-23.

5. "Aqueductal Stenosis". UCLA Neurosurgery. UCLA Health Available from: http://neurosurgery.ucla.edu/aqueductalstenosis. [Last accessed on 10 Mar 2016]

6. "Aqueductal Stenosis". Nervous System Diseases. Available from: http://www.nervous-system-diseases.com/aqueductalstenosis.html. [Last accessed on 10 Mar 2016].

7. Spennato P, S Tazi, O Bekaert, G Cinalli, P Decq. Endoscopic third ventriculostomy for idiopathic aqueductal stenosis. World Neurosurgery 2013;79(2 Suppl):S21,e13-20.

8. McMillan JJ, Williams B. Aqueduct stenosis. Case review and discussion. J Neurol Neurosurg Psychiatry 1977;40:521-32.

9. https://ryanakajoey.files.wordpress.com/2013/05/ventricles of the brain1352510262050.gif. [Last accessed on 10 Mar 2016].

10. Matys T, Horsburgh A, Kirollos RW, Massoud TF. The aqueduct of Sylvius: applied 3-T magnetic resonance imaging anatomy and morphometry with neuroendoscopic relevance. J Neurosurg 2013;73:132-40.

11. Frederik L Giesel, Anthony R Hart, Horst K Hahn, Emma Wignall, Fabian Rengier, Roland Talanow, et al. 3D reconstructions of the cerebral ventricles and volume quantification in children with brain malformations. Academic Radiol 2009;16:610-7.

12. Greitz D, Nordell B, Ericsson A, Ståhlberg F, Thomsen C. Notes on the driving forces of the CSF circulation with special emphasis on the piston action of the brain. Neuroradiology 1991;33(Suppl):178-81.

13. Amila T, Thunyaseth $\mathrm{S}$. Mathematical model for dynamics of CSF through the aqueduct of sylvia based on an analogy of arterial dilation and contraction. Int J Mining, Metallurgy Mechanical Engineering 2015;3:227-32.

14. G Soza, R Grosso, C Nimsky, P Hastreiter, R Fahlbusch, G Greiner. Determination of the elasticity parameters of brain tissue with combined simulation and registration. Int J Med Robotics Computer Assisted Surgery 2005;1:87-95.

15. Jeong Hyun Lee MD, Ho Kyu Lee MD, Jae Kyun Kim MD, Hyun Jeong Kim MD, Ji Kang Park MD, Choong Gon Choi MD. CSF flow quantification of the cerebral aqueduct in normal volunteers using phase-contrast cine MR imaging. Korean J Radiol 2004;5:81-6.

\section{How to cite this article}

- $\quad$ Thalakotunage AH, Thunyaseth Sethaput.Quantification of CSF velocity through the narrowest point in the aqueduct of Sylvia for normal and normal pressure hydrocephalus patient by CFD analysis. Int J Pharm Pharm Sci 2016;8 Suppl 2:52-55. 